\title{
Porcine In Vivo Validation of a Virtual Contrast Model: The Influence of Contrast Agent Properties and Vessel Flow Rates
}

\author{
(D)T.W. Peach, (D) Y. Ventikos, (D).V. Byrne, and (D) Z. You
}

\begin{abstract}
BACKGROUND AND PURPOSE: Accurately and efficiently modeling the transport of angiographic contrast currently offers the best method of verifying computational fluid dynamics simulations and, with it, progress toward the lofty goal of prediction of aneurysm treatment outcome a priori. This study specifically examines the influence of estimated flow rate and contrast properties on such in silico predictions of aneurysm contrast residence and decay.
\end{abstract}

MATERIALS AND METHODS: Four experimental sidewall aneurysms were created in swine, with aneurysm contrast flow patterns and decay rates observed under angiography. A simplified computational fluid dynamics model of the experimental aneurysm was constructed from 3D angiography and contrast residence predicted a priori. The relative influence of a number of estimated model parameters (contrast viscosity, contrast density, and blood flow rate) on contrast residence was then investigated with further simulations.

RESULTS: Contrast infiltration and washout pattern were accurately predicted by the a priori computational fluid dynamics model; however, the contrast decay rate was underestimated by $\sim 25 \%$. This error was attributed to the estimated parent vessel flow rate alone, and the effects of contrast viscosity and density on the decay rate were found to be inconsequential. A linear correlation between the parent vessel flow rate and the corresponding contrast decay rate was observed.

CONCLUSIONS: In experimental sidewall aneurysms, contrast fluid properties (viscosity and density) were shown to have a negligible effect on variation in the modeled contrast decay rate. A strong linear correlation was observed between parent vessel flow rate and contrast decay over a physiologically reasonable range of flow rates.

ABBREVIATIONS: CFD = computational fluid dynamics; $\mathrm{Q}=$ flow rate $(\mathrm{mL} / \mathrm{min}) ; \operatorname{Re}=$ Reynolds number

T he complex hemodynamic environment in and around a cerebral aneurysm has been linked to the initiation, development, and possible rupture of the lesion. ${ }^{1-3}$ Observing angiographic contrast in the dome of an aneurysm remains the primary means a clinician uses to obtain a high-resolution and real-time assessment of both the quantity and quality of aneurysmal blood flow. These in vivo observations may be complemented by computational fluid dynamics (CFD) simulations, which elucidate properties that cannot be measured clinically, such as wall shear

Received January 13, 2016; accepted after revision June 2.

From the Department of Mechanical Engineering (T.W.P., Y.V.), University College London, London, UK; Department of Neuroradiology (J.V.B.), John Radcliffe Hospital, Oxford, UK; and Department of Engineering Science (Z.Y.), University of Oxford, Oxford, UK.

This study was funded by a grant from the Wellcome Trust (WT 088877/Z/09Z).

Please address correspondence to $Y$. Ventikos, PhD, Department of Mechanical Engineering, University College London, London WCIE 7JE, UK; e-mail: y.ventikos@ucl.ac.uk

-- Indicates open access to non-subscribers at www.ajnr.org

http://dx.doi.org/10.3174/ajnr.A4884 stress, and provide a predictive capacity and the possibility of virtual treatment planning. ${ }^{4,5}$

The role of CFD in the field of neuroradiology is controversial and has become the focus of much fruitful discussion. ${ }^{6-8}$ A key point in this discussion is the need to bring the disciplines closer by combining the merits of computational and bench testing with those of animal models and clinical cases, to move toward a sophisticated model of intracranial aneurysm as a disease. ${ }^{9,10}$ Angiographic contrast transport is the only property common to both simulated hemodynamic measures and the complex biochemical environment that may be observed clinically. Hence, the need for accurately modeling contrast in silico is clear: CFD simulations may begin to be verified with the clinical criterion standard, and the quantification of aneurysm contrast/blood residence times has the potential to provide a direct link to models of intracranial aneurysm remodelling and thrombus formation. ${ }^{11-13}$

Implementations of such virtual contrast models, which approximate contrast as a dye-like scalar, are relatively simple. ${ }^{11,14-16}$ Although these simulations produce physically mean- 
ingful contrast washout behavior, inaccuracies remain that are attributed to not explicitly modeling contrast properties (density and viscosity) or variations in patient-specific vessel flow rates. ${ }^{14,15,17}$ Some models in the literature have shown very accurate replication of contrast infiltration and washout curves by directly matching properties estimated from angiography a posteriori (heart rate, flow rate, contrast bolus profile, and so forth). ${ }^{15}$ However, the clinical usefulness and predictive strength of such a result are limited.

This study was instead conducted by using a generalist approach; only the aneurysm and parent vessel 3D geometry were extracted from angiography with all other simulation parameters estimated. A range of parameters were modeled, including the parent vessel flow rate and both the viscosity and density of the angiographic contrast medium, with the relative influence of each parameter on contrast decay quantified. The surgically created aneurysm model, with known dimensions, allowed any variation in results due to angiographic reconstruction to be controlled for, unlike in previous studies. ${ }^{11,14-16}$ Such an approach makes advances toward accurate a priori prediction of contrast washout, requiring only a $3 \mathrm{D}$ reconstruction of the aneurysm in question, which may be obtained from any imaging technique.

\section{MATERIALS AND METHODS}

\section{Experimental Setup}

Experimental aneurysms were created in a porcine animal model by using 2 female Large White pigs of $60-65 \mathrm{~kg}$ and adopting the method described by German and Black. ${ }^{18}$ Each sidewall aneurysm was formed surgically by vein graft by using the jugular vein cut at $45^{\circ}$, forming an anastomosis on the common carotid artery with continuous $8 / 0$ stitching. One aneurysm was formed on each common carotid artery in both animals, with a total of 4 experimental aneurysms of near-identical size and shape. The common carotid artery was observed to have a diameter of approximately 4 $\mathrm{mm}$ in both animals, and the resulting sidewall aneurysms had a 5-mm diameter, 15-mm length, and 7-mm neck diameter. After creation, the aneurysms healed for 3 weeks to allow any local swelling to dissipate and for endothelialization at the anastomosis site to stabilize. Both animals were administered dual antiplatelet therapy following the surgical procedure.

Three weeks postcreation, each animal was catheterized via the femoral artery, and angiography was performed on an Innova 4100 system (GE Healthcare; Milwaukee, Wisconsin). 3D rotational angiography was used to accurately reconstruct the vessel and aneurysm geometry, while 2D angiography was used to verify the patency of each aneurysm and observe angiographic contrast washout in the aneurysm dome. A single series of 2D DSA acquisitions in the coronal plane was made for each of the 4 experimental aneurysms. Additionally, sagittal plane imaging was performed to verify complete and uniform contrast filling in each aneurysm. The $3 \mathrm{D}$ rotational runs were performed at a rotation speed of $20^{\circ}$ per second with a $20-\mathrm{mL}$ contrast injection delivered at $2 \mathrm{~mL} / \mathrm{s}$, and the $2 \mathrm{D}$ runs were conducted at 7.5 frames per second with a $10-\mathrm{mL}$ contrast injection delivered at $7.5 \mathrm{~mL} / \mathrm{s}$. In all imaging, the catheter tip was located $\sim 100 \mathrm{~mm}$ (25 vessel diameters) proximal to the aneurysm, and good contrast mixing was observed across the entire vessel lumen.
Ethics approval was obtained from the Oxford institutional ethics committee. Both animals were subsequently implanted with a flow-diverter device and formed part of a larger unrelated study, which is not reported in this article.

\section{Postprocessing}

DICOM postprocessing was completed in OsiriX Imaging Software, Version 7.5.1 (http://www.osirix-viewer.com), where 3D geometries were segmented and the resulting vascular surfaces were exported as STereoLithograph files. Good correspondence was seen among a number of metrics (vessel diameter, aneurysm diameter, aneurysm neck diameter, and aneurysm length) measured at both the surgical creation and on the $3 \mathrm{D}$ reconstruction created from angiography 3 weeks postcreation. Visually, the flow patterns among the 4 experimental aneurysms appeared identical, with contrast entering the aneurysm neck distally and circulating around the whole aneurysm dome, forming 1 large vortex.

The 2D DICOM images were exported and segmented in Matlab (MathWorks, Natick, Massachusetts). A segmentation window isolating the aneurysm dome from the parent vessel was created manually for each experimental aneurysm, and the mean gray-scale value in the aneurysm dome was calculated for each DICOM in the time-series.

\section{Experimental Calibration}

To verify the direct correspondence between pixel gray-scale value and contrast concentration, we performed a brief calibration experiment. A number of $10-\mathrm{mL}$ syringes were filled with a mixture of saline and contrast medium in varying concentrations ( $0 \%-50 \%$ contrast by volume) and were imaged by using a standard neck/cerebral DSA protocol. After we subtracted background values, the mean gray-scale value of each syringe was found to vary linearly with contrast concentration.

\section{CFD Simulations}

Given the similarity in vessel size, aneurysm size, and aneurysm flow pattern among the 4 experimental aneurysms and in the interest of increasing computational efficiency, the $3 \mathrm{D}$ geometry of only 1 aneurysm was selected for the computational study. The aneurysm geometry was imported into CFD-VisCART (ESI Group, Paris, France) and meshed; unstructured meshing was completed with a minimum mesh density of 4000 elements $/ \mathrm{mm}^{3}$, resulting in a total mesh size of 4,290,000 elements.

Contrast was modeled computationally as an independent scalar quantity introduced at the simulation inlet and transported in the bulk flow, similar to dye particles. Although this method is a major simplification of what is, in reality, a 2 -fluid mixing problem, the reduction in both computational cost and the complexity required to characterize true 2 -fluid flow are considerable. The modeled contrast does not alter the simulated blood density or viscosity and has a diffusivity in blood of $1.0 \times 10^{-7} \mathrm{~mm}^{2} / \mathrm{s}$, an order of magnitude estimate based on the diffusivity of tracer particles in blood. ${ }^{19}$ A uniform contrast bolus was prescribed at the geometry inlet with a duration of 1 cardiac cycle. Thus, the total contrast transport seen virtually can be considered analogous to the experimental transport averaged over the cardiac cycle.

The computational mesh was imported into the multiphysics 


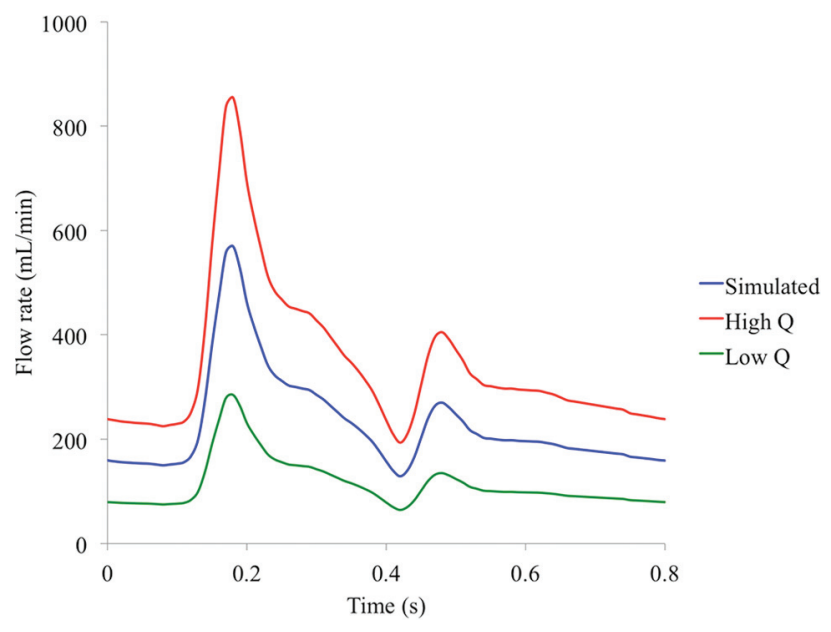

FIG 1. Parent vessel flow rate profiles based on the human ICA.

suite CFD-ACE+ (ESI Group). Blood flow was modeled as an incompressible fluid with unsteady 3D Navier-Stokes governing equations, with second-order interpolations in space and time, a time-step of 0.01 second, a rigid arterial wall, a fluid density of $1000 \mathrm{~kg} / \mathrm{m}^{3}$, and a dynamic viscosity of $0.004 \mathrm{~Pa} \times \mathrm{s}$. We adopted a Newtonian fluid model, assuming that non-Newtonian flow effects are small in both the aneurysm dome and arteries with a diameter of $>0.5 \mathrm{~mm} \cdot{ }^{20,21}$ We ignored gravitational effects, assuming that the experimental aneurysms remained located, as created, in the approximate horizontal (coronal) plane.

The transient simulation was run with a time-varying inflow based on the profile of flow in the human ICA, derived from a 1D model of the vasculature, as depicted in Fig $1 .^{22}$ Because an accurate profile of flow in a porcine common carotid artery was not available to the authors, we concluded that the similarity in flow rate, artery diameter, and distance from the heart between the porcine common carotid artery and the human ICA made this assumption reasonable. This assumption is interrogated further in the discussion that follows. A radially symmetric inlet velocity profile was prescribed at the inlet and scaled to give a mean flow rate of $230 \mathrm{~mL} / \mathrm{min}$.

The simulation was run on thirty-two $2.93-\mathrm{GHz}$ cores, with each time-step converging to 5 orders of magnitude residual reduction in around 50-100 iterations in a typical solution time of 30 minutes per time-step. The contrast bolus of 0.8 -second duration was introduced after 2 full cardiac cycles ( 1.60 seconds real time) to reduce the influence of initial transient flow effects. The simulation was run for a further 4 cardiac cycles after the contrast bolus was first introduced, giving a total simulation time of 6 cardiac cycles ( 4.80 seconds real time). The absolute value of contrast residing in the aneurysm dome of the simulated geometry was then exported at every fourth time-step.

Virtual angiography was generated by aligning the simulation geometry into the same plane as that seen experimentally before the simulated contrast concentration distribution was then discretized into a $100 \times 150$ grid of pixels (approximately matching the experimental imaging resolution of $0.2 \times 0.2 \mathrm{~mm}$ ).
Table 1: Contrast media properties

\begin{tabular}{lcc}
\hline Contrast Agent & Density & Viscosity \\
\hline Omnipaque 180 & $1164 \mathrm{~kg} / \mathrm{m}^{3}$ & $0.0015 \mathrm{~Pa} \times \mathrm{s}$ \\
Omnipaque 350 & $1410 \mathrm{~kg} / \mathrm{m}^{3}$ & $0.0204 \mathrm{~Pa} \times \mathrm{s}$ \\
\hline
\end{tabular}

Table 2: Contrast-blood mixture properties

\begin{tabular}{lcrc}
\hline & & & Modified \\
& Density & \multicolumn{1}{c}{ Viscosity } & Re \\
\hline High Re (Omnipaque 180) & $1080 \mathrm{~kg} / \mathrm{m}^{3}$ & $0.00275 \mathrm{~Pa} \times \mathrm{s}$ & $+70 \%$ \\
Low Re (Omnipaque 350) & $1200 \mathrm{~kg} / \mathrm{m}^{3}$ & $0.0122 \mathrm{~Pa} \times \mathrm{s}$ & $-40 \%$ \\
\hline
\end{tabular}

\section{Accounting for Contrast Properties}

A second set of simulations was conducted to quantify the influence of contrast properties. In the absence of a true 2-fluid model, flow effects arising from the local gradient in viscosity and density between blood and contrast could not be modeled explicitly. Instead, we ran simulations, assuming a working fluid equivalent to a perfect 50:50 mixture of blood and contrast, which was transported though the geometry for the entire simulation (ie, before, during, and after the virtual contrast bolus was introduced).

In an attempt to quantify the overall influence of contrast properties, 2 media were chosen that represented the greatest overall change in fluid properties. Specifically, contrast media with the highest and lowest ratios of density to viscosity were chosen (as summarized in Table 1), which correspondingly increase and decrease the simulation Reynolds number $(\mathrm{Re})$ to the greatest degree.

The density, viscosity, and equivalent change in inlet Reynolds number for the simulations incorporating the properties of each contrast agent (assuming a 50:50 mix of contrast and blood) are summarized in Table 2. For comparison, the equivalent mixture of blood and the contrast agent used experimentally (iohexol, Omnipaque 240; GE Healthcare, Piscataway, New Jersey) would result in an effective increase in the Re of $23 \%$. Both simulations (high Re and low $\mathrm{Re}$ ) were run with the same parameters and postprocessed in the same manner as described in the previous section.

\section{Accounting for Parent Vessel Flow Rate}

Approximating the average and instantaneous parent vessel flow rate is an additional source of error in simulated contrast washout behavior that is often reported in the literature. To quantify this variation, we conducted a third and final set of CFD simulations with altered mean parent vessel flow rates corresponding to a $50 \%$ increase (345 $\mathrm{mL} / \mathrm{min})$ and $50 \%$ decrease $(115 \mathrm{~mL} / \mathrm{min})$ compared with the mean human ICA rate $(230 \mathrm{~mL} / \mathrm{min})$ used in previous simulations. The overall shape of the flow profile in time was unaltered and was only scaled to achieve an altered flow rate, as indicated in Fig 1.

The 50\% increased flow rate was chosen as an approximation to the flow rate of a human common carotid artery, which could also be considered an upper estimate of the flow rate in the experimental porcine common carotid artery. The 50\% decreased flow rate was selected as an order-of-magnitude estimate based on previous experiments conducted by the authors, which quantified flow rate reduction in a vessel cross-section partially occluded by a catheter lumen. Hence, it was assumed that the range of mean flow rates considered (115-345 $\mathrm{mL} / \mathrm{min}$ ) encompassed that likely to be seen experimentally. Both simulations with altered flow 
rate (Q) (low Q and high Q) were run with solver settings and postprocessing procedures identical to simulations discussed previously.

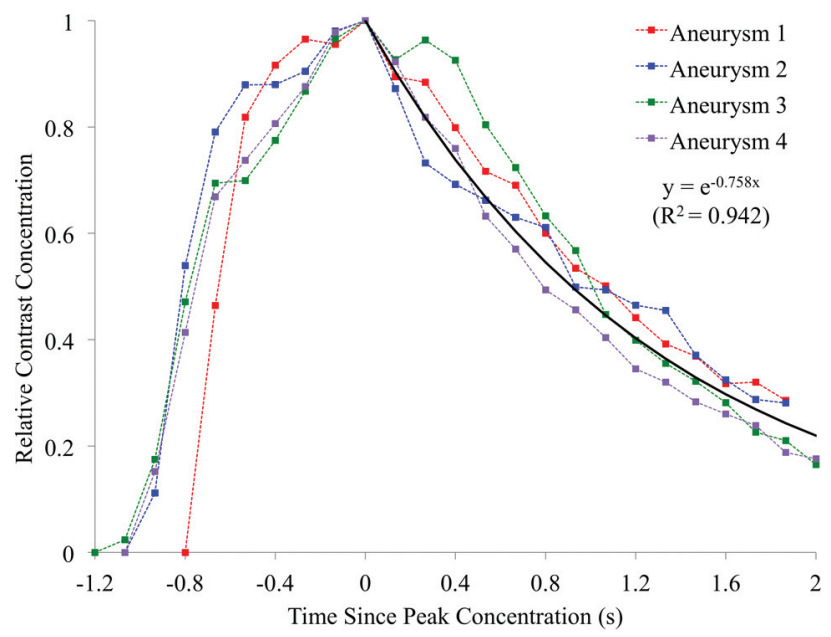

FIG 2. Experimental contrast residence curves for all 4 experimental aneurysms and the fitted exponential decay curve.

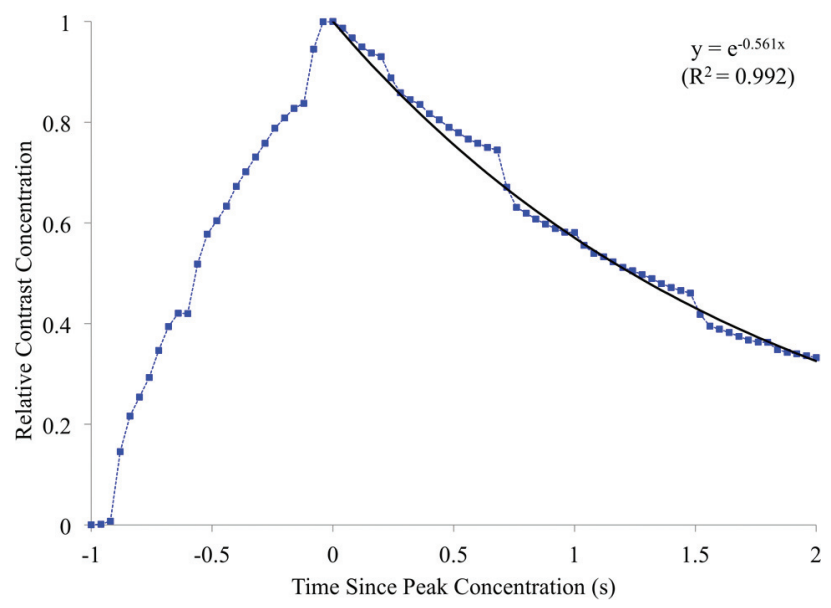

FIG 3. Simulated contrast residence curve and the fitted exponential decay curve.
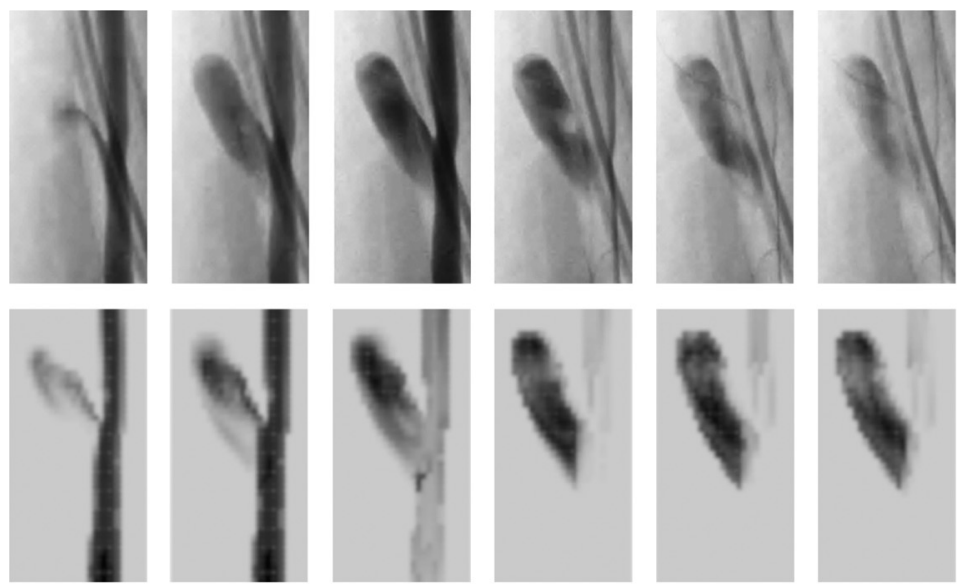

$-0.8$

$-0.4$
0.4

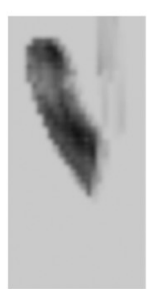

1.2

FIG 4. Experimental (upper) and virtual (lower) DSA. Images are separated by 0.40 -second intervals and aligned at 0.0 seconds to peak aneurysm contrast concentration.

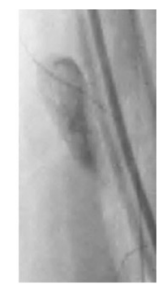

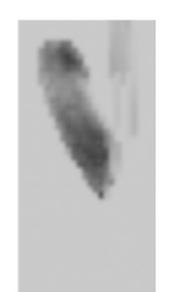

1.6

\section{Inferring Experimental Flow Rate}

Finally, by tracking the contrast-front transport in consecutive DICOM images, we calculated an estimate of the true experimental parent vessel flow rate, assuming a Poiseuille velocity profile. The velocity of the contrast-front was recorded for 2 consecutive 0.133 second intervals in each experimental aneurysm, which resulted in 8 separate estimates of the instantaneous parent vessel flow rate.

\section{RESULTS}

Contrast concentration, averaged over the aneurysm dome, for Coxperimental aneurysm is summarized in Fig 2. The contrast curves are aligned to peak relative contrast concentration, at $\mathrm{t}=0$ conds. Assuming a capacitor-based model of contrast residence, fitted an exponential decay curve to the decay portion of the erimental data with a decay constant of 0.758 second $^{-1}$. Simed contrast residence is shown in Fig 3, with a corresponding ential curve fitted and a decay constant of 0.561 second $^{-1}$ rimental and virtual angiography is shown in Fig 4, where the convention of aligning the peak contrast concentration to

The contrast decay curves for the simulations conducted at altered Reynolds numbers and parent vessel flow rates are displayed in Figs 5 and 6, respectively. These decay curves are plotted alongside both the initial simulated contrast concentration and the experimental results. The corresponding exponential decay constants for the experimental results and each of the 5 simulaline fitted, assuming direct proportionality.

Finally, the 8 instantaneous parent vessel flow rates that were extracted from the experimental angiography are found to correspond to a mean parent vessel flow rate of $296 \mathrm{~mL} / \mathrm{min}$ with a range of $202-516 \mathrm{~mL} / \mathrm{min}$.

\section{DISCUSSION}

The global contrast transport in the experimental aneurysm dome is well-described by the exponential decay model proposed in Fig 2 , with little variation seen across all 4 experimental aneurysms. In agreement with the observations of others in the literature, the modeling of contrast agent in this study as an independent scalar transported in simulated fluid flow accurately replicates the behavior seen experimentally. ${ }^{14-16}$

The overall contrast infiltration and washout patterns are similar for the experimental and simulated cases (Figs 2 and 3), but the simulated contrast decay constant is $26 \%$ lower than the experimental value. As such, after 2.0 seconds, approximately $25 \%$ of the total contrast volume remains in the aneurysm in the experimental case, but $35 \%$ of the simulated contrast remains. This underestimation of the simulated contrast decay constant $\left(0.560\right.$ second $\left.^{-1}\right)$ is beyond the range of scatter seen in the experimental 


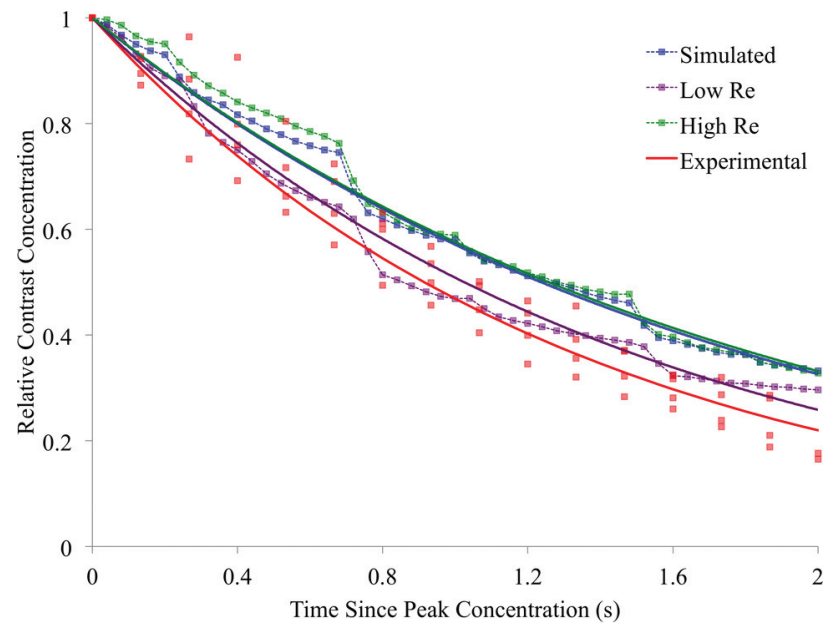

FIG 5. Simulated and experimental contrast decay incorporating variation in simulated contrast density and viscosity.

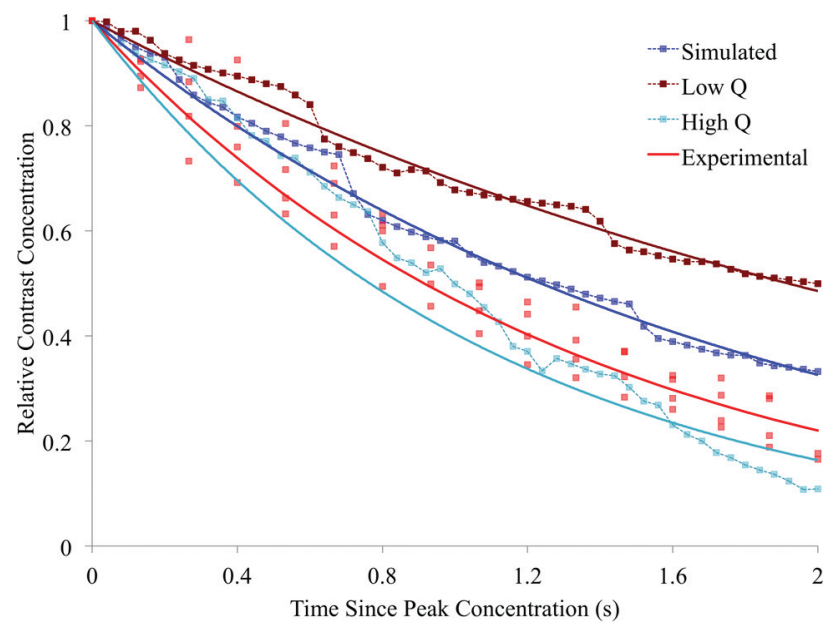

FIG 6. Simulated and experimental contrast decay incorporating variation in the simulated parent vessel flow rate.

Table 3: Summary of experimental and simulated contrast decay constants

\begin{tabular}{lc}
\hline & Contrast Decay Constant $^{-1}$ \\
\hline Experimental & 0.758 second $^{-1}$ \\
Initial simulation & 0.560 second $^{-1}$ \\
High Re simulation & 0.552 second $^{-1}$ \\
Low Re simulation & 0.677 second $^{-1}$ \\
High Q simulation & 0.903 second $^{-1}$ \\
Low Q simulation $^{-1}$ & 0.362 second $^{-1}$ \\
\hline
\end{tabular}

data, where curves fitted to individual experimental aneurysm decay traces show decay constants of $0.68-0.859$ second $^{-1}$. The physical distribution of contrast within the aneurysm dome is wellpredicted by the simulation, with similar areas of infiltration, circulation, and washout visible in the experimental and simulated angiography of Fig 4. However, the lower rate of contrast decay in the simulated case results in increased aneurysm contrast residence and a noticeably darker angiogram.

The range in simulated contrast decay constant seen over the high Re and low Re simulations is small and of the order of the scatter seen in the experimental data in Fig 5. Additionally, the range of decay constants across the 2 simulations $\left(0.552-0.677\right.$ second $\left.^{-1}\right)$ does not

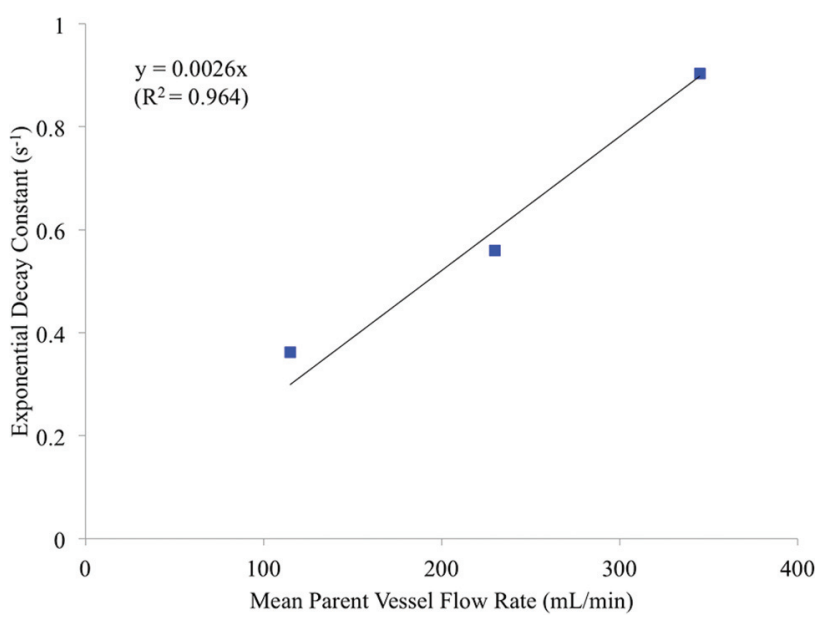

FIG 7. Simulated contrast decay constant and mean parent vessel flow rate.

encompass the experimental value $\left(0.758\right.$ second $\left.^{-1}\right)$. Such a result suggests that the differences between blood and contrast fluid properties alone are unlikely to account for the discrepancy in the predicted contrast decay rate seen in the original simulation (Fig 3). This assumption is further reinforced by the highly asymmetric spread of the high Re and low Re curves in Fig 5, with no discernable difference between the initial and high Re simulations and the experimental contrast agent used, corresponding to a slight increase (23\%) in the simulation Reynolds number.

As indicated in both Fig 6 and Table 3, the range in decay constant seen for the high Q and low Q simulations is both more substantial than the range seen in Fig 5 and encompasses the decay seen experimentally. Hence, a discrepancy between the experimental flow rate and the flow rate assumed a priori in the original CFD simulation is the most likely cause of the poor experimental contrast decay rate prediction.

The spread of the high Q and low Q simulation decay curves about the original simulation in Fig 6 is relatively symmetric, unlike the previous simulations of Fig 5. However, the high Q contrast curve is not as well-approximated to an exponential decay as the other simulation results; this outcome is likely due to the low degree of flow recirculation observed in the simulation, where flow streamlines traced the perimeter of the aneurysm dome before immediately exiting.

From the decay curves in Fig 6, it appears that a simulation adopting a parent vessel flow rate between the mean human ICA rate of the original simulation and the mean human common carotid artery rate of the high Q case would result in a contrast decay curve similar to that obtained experimentally. When the simulation flow rate and contrast decay rate for the high Q, low $\mathrm{Q}$, and original simulations are plotted in Fig 7, a linear and directly proportional trend line may be fitted to the data. Thus, if one assumes this linear relationship between the decay and flow rates and an experimental cardiac profile similar to that in the human ICA/common carotid artery, a simulated mean parent vessel flow rate of approximately $290 \mathrm{~mL} / \mathrm{min}$ would mimic the contrast decay rate of the in vivo model $\left(0.758\right.$ second $\left.^{-1}\right)$. Such a mean flow rate is both reasonable and only $2 \%$ lower than the mean flow rate of $296 \mathrm{~mL} / \mathrm{min}$ that was estimated from experimental angiography. 


\section{Study Limitations}

As previously discussed, the simplified contrast model implemented does not explicitly model the mixing of 2 fluids; hence, local gradients of contrast and blood fluid properties are not captured. However, given the rapid and fairly complete degree of blood and contrast mixing seen experimentally and the fundamental design of the contrast medium being highly diffusive, such a phenomenon is likely insignificant.

The experimental 2D DSA frame rate of 7.5 frames per second was chosen to approximately match the temporal resolution frequently used in the clinical setting. Although some flow effects may not be fully resolved temporally at such a frame rate, the relatively smooth curves and lack of any aliasing in Fig 2 suggest that the global aneurysm flow effects are adequately resolved. Additionally, the flow features observed in the simulated results (with an effective frame rate of 100 frames per second) did not show any high-frequency variations.

Over the range of flow rates considered $(115-345 \mathrm{~mL} / \mathrm{min})$, there appears to be a simple linear relation between the parent vessel flow rate and aneurysm contrast decay rate. However, it is unclear whether a similar relationship would hold for other aneurysm geometries under similar flow rates. More generally, this study focused only on a sidewall aneurysm model, so any conclusions are unlikely to hold true in the drastically different flow environment of bifurcation aneurysms.

Finally, this study only focused on the global contrast concentration within the aneurysm dome. Local variation in contrast concentration, both in and out of the 2D DSA plane, was not quantified for simplicity and brevity, but a strong qualitative similarity was seen between experimental and simulated local contrast concentration patterns. The use of $4 \mathrm{D}$ angiographic technology in any future study would allow the time-varying 3D distribution of contrast in the aneurysm dome to be fully captured and the effect of contrast and flow properties on contrast distributions to be interrogated more completely.

\section{CONCLUSIONS}

Good correspondence was seen between a CFD contrast transport model and experimental results. Aneurysm flow patterns and contrast decay mechanisms were accurately predicted by simulation and may have the potential to offer clinical insight in treatment planning.

Modeled contrast fluid properties (viscosity and density) were shown to have a negligible effect on the aneurysm contrast decay rate, compared with assuming properties identical to a Newtonian blood model. Mismatch between the experimental and simulated contrast decay rate was attributed to an initial underestimate of the simulated parent vessel flow rate, later verified by angiography. This influence of patient-specific vessel flow rates remains a major challenge to a priori prediction of aneurysm contrast residence and treatment outcomes.

\section{ACKNOWLEDGMENTS}

All authors would like to thank Dr M. Megahed of the ESI Group for allowing the use of the CFD-ACE suite.
Disclosures: Thomas Peach, Yiannis Ventikos, Zhong You-RELATED: Grant: Wellcome Trust/Engineering and Physical Sciences Research Council (grant WT 088877/ Z/09Z).* James Byrne—RELATED: Grant: Wellcome Trust (grant WT 088877/Z/ 09Z);* Support for Travel to Meetings for the Study or Other Purposes: Wellcome Trust (grant WT 088877/Z/09Z). *Money paid to the institution.

\section{REFERENCES}

1. Brisman JL, Song JK, Newell DW. Cerebral aneurysms. N Engl J Med 2006;355:928-39 CrossRef Medline

2. Sforza DM, Putman CM, Cebral JR. Hemodynamics of cerebral aneurysms. Annu Rev Fluid Mech 2009;41:91-107 CrossRef Medline

3. Xu J, Yu Y, Wu X, et al. Morphological and hemodynamic analysis of mirror posterior communicating artery aneurysms. PLoS One 2013;8:e55413 CrossRef Medline

4. Steinman D, Milner JS, Norley CJ, et al. Image-based computational simulation of flow dynamics in a giant intracranial aneurysm. AJNR Am J Neuroradiol 2003;24:559-66 Medline

5. Chung B, Cebral JR. CFD for evaluation and treatment planning of aneurysms: review of proposed clinical uses and their challenges. Ann Biomed Eng 2015;43:122-38 CrossRef Medline

6. Kallmes DF. Point: CFD-computational fluid dynamics or confounding factor dissemination. AJNR Am J Neuroradiol. 2012;33: 395-96 CrossRef Medline

7. Cebral JR, Meng H. Counterpoint: realizing the clinical utility of computational fluid dynamics - closing the gap. AJNR Am J Neuroradiol 2012;33:396-98 CrossRef Medline

8. Ventikos Y. Resolving the issue of resolution. AJNR Am J Neuroradiol 2014;35:544-45 CrossRef Medline

9. Robertson AM, Watton PN. Computational fluid dynamics in aneurysm research: critical reflections, future directions. AJNR Am J Neuroradiol 2012;33:992-95 CrossRef Medline

10. Strother CM, Jiang J. Intracranial aneurysms, cancer, $\mathbf{x}$-rays, and computational fluid dynamics. AJNR Am J Neuroradiol 2012;33: 991-92 CrossRef Medline

11. Sadasivan C, Cesar L, Seong J, et al. An original flow diversion device for the treatment of intracranial aneurysms: evaluation in the rabbit elastase-induced model. Stroke 2009;40:952-58 CrossRef Medline

12. Cebral JR, Vazquez M, Sforza DM, et al. Analysis of hemodynamics and wall mechanics at sites of cerebral aneurysm rupture. J Neurointerv 2015;7:530-36 CrossRef Medline

13. Gester K, Lüchtefeld I, Büsen M, et al. In vitro evaluation of intra-aneurysmal, flow-diverter-induced thrombus formation: a feasibility study. AJNR Am J Neuroradiol 2016;37:490-96 CrossRef Medline

14. Ford MD, Stuhne GR, Nikolov HN, et al. Virtual angiography for visualization and validation of computational models of aneurysm hemodynamics. IEEE Trans Med Imaging 2005;24:1586-92 CrossRef Medline

15. Endres J, Kowarschik M, Redel T, et al. A workflow for patient-individualized virtual angiogram generation based on CFD simulation. Comput Math Methods Med 2012;2012:306765 CrossRef Medline

16. Cebral JR, Pergolizzi RS Jr, Putman CM. Computational fluid dynamics modeling of intracranial aneurysms: qualitative comparison with cerebral angiography. Acad Radiol 2007;14:804-13 CrossRef Medline

17. Wang ZJ, Hoffmann KR, Wang Z, et al. Contrast settling in cerebral aneurysm angiography. Phys Med Biol 2005;50:3171-81 CrossRef Medline

18. German W, Black S. Experimental production of carotid aneurysms. N Engl J Med 1954;250:104-06 CrossRef Medline

19. Saadatmand M, Ishikawa T, Matsuki N, et al. Fluid particle diffusion through high-hematocrit blood flow within a capillary tube. J Biomech 2011;44:170-75 CrossRef Medline

20. Perktold K, Resch M, Florian H. Pulsatile non-Newtonian flow characteristics in a three-dimensional human carotid bifurcation model. J Biomech Eng 1991;113:464-75 CrossRef Medline

21. Valencia AA, Guzmán AM, Finol EA, et al. Blood flow dynamics in saccular aneurysm models of the basilar artery. J Biomech Eng 2006; 128:516-26 CrossRef Medline

22. Reymond P, Vardoulis O, Stergiopulos N. Generic and patient-specific models of the arterial tree. J Clin Monit Comput 2012;26:375-82 CrossRef Medline 